\title{
Bridging a translational gap: using machine learning to improve the prediction of PTSD
}

\author{
Karen-Inge Karstoft ${ }^{2 *}$, Isaac R Galatzer-Levy ${ }^{1}$, Alexander Statnikov ${ }^{3,4}$, Zhiguo Li ${ }^{3}$, Arieh Y Shalev ${ }^{1}$ and For members \\ of the Jerusalem Trauma Outreach and Prevention Study (J-TOPS) group
}

\begin{abstract}
Background: Predicting Posttraumatic Stress Disorder (PTSD) is a pre-requisite for targeted prevention. Current research has identified group-level risk-indicators, many of which (e.g., head trauma, receiving opiates) concern but a subset of survivors. Identifying interchangeable sets of risk indicators may increase the efficiency of early risk assessment. The study goal is to use supervised machine learning (ML) to uncover interchangeable, maximally predictive combinations of early risk indicators.

Methods: Data variables (features) reflecting event characteristics, emergency department (ED) records and early symptoms were collected in 957 trauma survivors within ten days of ED admission, and used to predict PTSD symptom trajectories during the following fifteen months. A Target Information Equivalence Algorithm (TIE*) identified all minimal sets of features (Markov Boundaries; MBs) that maximized the prediction of a non-remitting PTSD symptom trajectory when integrated in a support vector machine (SVM). The predictive accuracy of each set of predictors was evaluated in a repeated 10-fold cross-validation and expressed as average area under the Receiver Operating Characteristics curve (AUC) for all validation trials.
\end{abstract}

Results: The average number of MBs per cross validation was $800 . \mathrm{MBs}^{\prime}$ mean AUC was 0.75 (95\% range: 0.67-0.80). The average number of features per MB was 18 (range: 12-32) with 13 features present in over $75 \%$ of the sets.

Conclusions: Our findings support the hypothesized existence of multiple and interchangeable sets of risk indicators that equally and exhaustively predict non-remitting PTSD. ML's ability to increase prediction versatility is a promising step towards developing algorithmic, knowledge-based, personalized prediction of post-traumatic psychopathology.

Keywords: Posttraumatic Stress Disorder (PTSD), Machine learning, Early prediction, Risk factors, Markov boundary feature selection, Support vector machines

\section{Background}

The early identification of individuals at risk for developing posttraumatic stress disorder (PTSD) is a major clinical and public health challenge, which many studies have attempted to address (for meta-analyses, see Brewin et al. [1] and Ozer et al. [2]). Currently-identified risk indicators include event characteristics [3], peri-traumatic responses [4-6], early symptoms [7-10], early physiological and neuroendocrine responses [11], gene expression profiles [12] and recovery environment factors [13]. Together, current findings suggest that PTSD is associated

\footnotetext{
* Correspondence: kikarstoft@health.sdu.dk

${ }^{2}$ Research and Knowledge Centre, Danish Veteran Centre, Garnisonen 1, 4100 Ringsted, Denmark

Full list of author information is available at the end of the article
}

with an array of multimodal risk indicators, many of which are observable shortly after trauma exposure. Despite these findings, research to date has failed to reveal clinically useful, personalized predictors.

This translational gap has several reasons: Previous studies have identified risk indicators at the group level, thereby overlooking within-group heterogeneities and distinct individual paths to PTSD that emanate from the disorder's complex multi-causal etiology [14]. Based on the general linear model, statistical methods used were not optimally suited to explore the complex interactions between linear, non-linear or non-normally distributed risk indicators encountered during trauma and its early aftermath [15]. 
Additionally, within the inherently complex and multimodal matrix of emerging post-traumatic morbidity, the relative contribution of any risk-indicator is necessarily context-dependent and thus does not directly translate across traumatic events and individuals exposed (e.g., female gender increases the likelihood of PTSD among survivors of physical assault, but not in accidents victims [3]). Consequently proper risk assessment defies simple computation and requires knowledge-based, rule driven expert systems.

Importantly, many of the currently known risk indicators may not be present, or not captured in every exposed individual. For example, elevated heart rate response to traumatic events, whilst repeatedly associated with subsequent PTSD $[5,16]$ is only recorded in survivors who are brought to medical attention. Other known risk moderators, such as head injury $[17,18]$ or receiving opiates following injury [19] similarly concern a subset of survivors.

To overcome these limitations, forecasting methods of PTSD must accommodate multiple combinations of risk indicators, account for partially available information and use prior knowledge to adjust the relative weights of putative predictors. The goal of the present work is to address the first requirement, namely, evaluate the use of multiple combinations or 'sets' of data items to predict post-traumatic morbidity, assess the accuracy the predictive power made from such sets.

To accomplish this goal, this work applied machine learning (ML, see glossary in Additional file 1) modeling to a large longitudinal dataset and evaluated the method's ability to identify multiple, equally predictive sets of variables. ML based forecasting models can accommodate different configurations of predictive features, integrate multi-modal variables, assign context-driven weights to putative predictors and identify multiple sets of variables that exhaust the predictive power of available features [20-22].

In a previous study [23], we evaluated the ability of ML-based feature-selection algorithm to extract one set of early risk indicators. We also compared various ML classification algorithms and evaluated predictability of two outcome configurations: PTSD at end point and membership in a non-remitting PTSD symptom trajectory. That study demonstrated that data representing the traumatic event and subsequent ED admission (e.g., head injury, length of stay in the ED) improves the prediction from early symptoms. It showed equal performance of six classification algorithms and better predictability of the non-remitting PTSD symptom trajectory relative to diagnostic status. Building on these findings, this work uses support vector machines (SVMs) as its classification algorithm and a non-remitting PTSD symptom trajectory as the predicted outcome. This work, therefore, expands the scope of the previous study by evaluating multiple, equivalent, maximally predictive sets of early features.
Specifically, we applied a Target Information Equivalence algorithm (TIE-star or TIE*; Figure 1) to uncover all compact non-redundant sets of items that maximize the prediction of non-remitting PTSD symptom trajectory [20,4]. We then evaluated the accuracy of prediction from each of these sets using support vector machines (SVMs [24]).

\section{Methods}

\section{Participants and Procedures}

This study used data collected for the Jerusalem Trauma Outreach and Prevention Study (J-TOPS [25-27]; ClinicalTrial.Gov identifier: NCT0014690). Participants were adults (age: 18-70) consecutively admitted to ED following potentially traumatic events (PTEs). Participants provided oral and written informed consent for, respectively, telephone based and face-to-face phases of the study. The study's procedures were approved and monitored by the Hadassah University Hospital's Institutional Review Board.

Eligible participants $(n=4,743)$ were screened by short telephone interviews, and those with confirmed PTEs as per DSM-IV PTSD criteria A1 and A2 $(n=1,996)$ received structured, telephone-based interviews ten days (exactly $9.61 \pm 3.91$ days) after trauma exposure. Telephone based assessments were repeated seven months (n $=1,784)$ and fifteen months $(n=1,022)$ following ED admission. Participants with acute PTSD symptoms in the first assessment $(n=1,502)$ were additionally invited for clinical interviews, which $\mathrm{n}=756$ attended, $19.80 \pm$ 5.17 days after ED admission. Participants of the first clinical assessment were re-evaluated five months after the traumatic event $(144.1 \pm 35.2$ days; $\mathrm{n}=604)$. For detailed procedures, see Shalev et al. [27].

For the purpose of this study, we included individuals who had initial data available at ten days and at least two additional time points $(\mathrm{n}=957)$. Participants included in this study did not differ from those who were not included in gender distribution, age, general distress, initial PTSD symptoms and the frequency of exposure to new traumatic events during the study [24].

\section{Instruments}

Sixty-eight data items (features) were recorded during survivors' ED admission and in the first telephone interviews. ED features included demographics, trauma types (traffic accidents, work accidents, terrorist attacks and other incidents), loss of consciousness during the traumatic incident, head injury, whiplash injury, blood pressure, pulse, perceived pain (a 1-10 pain intensity scale), prescribed analgesics and duration of ED admissions. Telephone interview features included individual items and total scores of the PTSD symptom scale (PSS) [28], the Kessler-6 (K6), [29] the Acute Stress Disorder Scale (ASDS) [30] and the Clinical Global Impression instrument (CGI) [31] which both participants and 


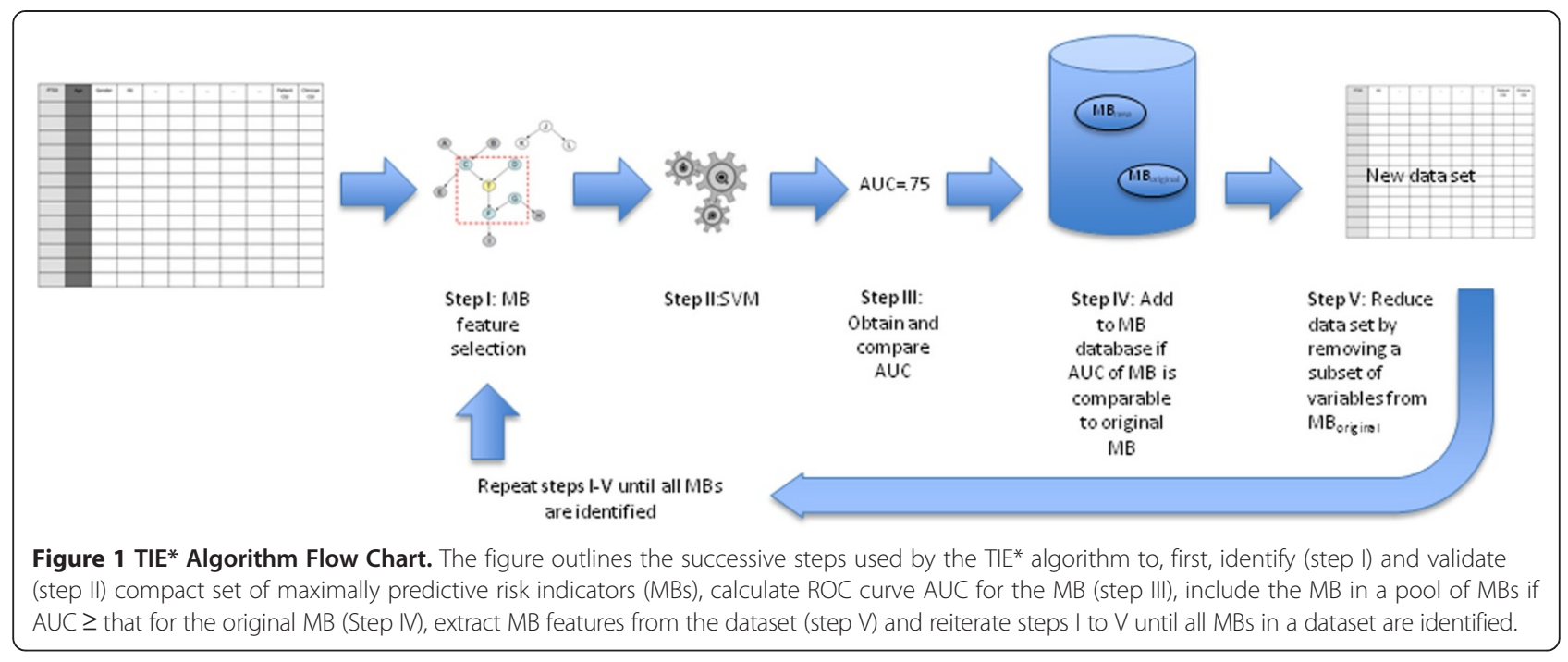

interviewers completed. They also included single items reflecting the four dimensions of the Posttraumatic Cognition Inventory (PTCI [32]: (counting on others, counting on oneself, dangerousness of the world, and self-blame) and four coping efficacy items [33] (sustained task performance, capacity for rewarding interpersonal communication, controllability of emotions, and positive self-perception), as well as participants' expressed need for help, perceived social support, and perceived fearfulness and threat embedded in the traumatic event.

\section{Modeling Approach Outcome Measure}

The study's main outcome measure was membership (yes/no) in a non-remitting PTSD symptom trajectory as defined in a previous LGMM-based study of this sample [25]. Studies have shown that the alternative outcome, PTSD diagnostic status, is unstable, fluctuates with time [34] and can be met with various degrees of symptom severity. In this dataset, the non-remitting PTSD symptom trajectory was not affected by treatment received and, as mentioned above, was better predicted than end point PTSD status [23].

\section{Machine learning approach}

Identification of risk indicators sets (MBs, Figure 1) To identify all compact sets of variables with optimal predictive accuracy, we applied the TIE* (Target Information Equivalence - Star) algorithm [20]. The TIE* algorithm: (i) Identifies a minimal set of variables that render all other predictors non-significant in relation to the outcome (a 'Markov Boundary' or, MB) and evaluates the accuracy of prediction using SVM, (ii) removes one or more of the obtained $\mathrm{MB}$ features from the data set, and repeats the analyses to identify a new $M B$ in the reduced data set, (iii) determines the accuracy of the new MB by feeding it in to a Support Vector Machine, and keeps the new MB if its predictive accuracy is statistically comparable to that of the original $\mathrm{MB}$, and (iv) reiterates steps (ii) and (iii) until all $M B S$ that provide equivalent predictive accuracy have been identified (Figure 1). The TIE* has been validated in previous studies [20]. The MB identification processes used in the TIE* are available in the Causal Explorer toolkit [35]. The SVM algorithm used is available at LibSVM [36].

Cross-validation procedure To test the robustness of predictors, we subsequently applied the TIE* procedure in a 10-fold cross-validation, in which participants are randomly split into ten non-overlapping subsets containing approximately the same number of cases and non-cases (patients following a non-remitting and a remitting course of PTSD, respectively). The classification algorithm is trained in nine of these ten data subsets, and subsequently (and independently) tested in the remaining tenth subset. This procedure is repeated iteratively, resulting in each of the ten data subsets being used once for testing of the model. We repeated the 10 -fold cross validation procedure 10 times to reduce splitting variance, resulting in a total of 100 repetitions of training and testing. Predictive accuracy was expressed as the mean accuracy obtained from SVM applied across all cross-validation runs. The frequency of features' presence across MBs was calculated as a measure of their predictive 'robustness'.

\section{Accuracy metric}

We estimated predictive accuracy using Area Under the ROC Curve (AUC). The ROC curve is a plot of the sensitivity versus 1 -specificity of a classification system, 
and measures the accuracy of that system, which can then be directly compared to that of another system [37]. To further investigate the accuracy, we also computed average sensitivity and specificity for various thresholds.

\section{Results}

Identification of MBs in full data set

Before cross-validation, the TIE* algorithm applied to the full data set $(\mathrm{N}=957)$ identified 789 distinct MBs. The average number of data items per MB was 18 (range 15-29). Thirty-four items participated in at least one MB.

\section{Cross-validation of MBs}

The average number of MBs identified in the repeated cross-validations was 800 . The average number of features per MB was 18 (range 12-32). Forty-seven features participated in at least one MB. Thirteen features participate in over $75 \%$ of all MBs (see Figure 2). The consistently predictive features include age, time in the ED, head injury, perceived ED pain, patient and clinician's clinical global impression, total PSS and K6 scores, reporting nightmares, concentration problems, feeling worthless, wanting help, and quality of social support. The average predictive accuracy of all MBs was within an acceptable range (AUC $=.75 ; 95 \%$ range $=0.67-0.80$ ).

\section{Discussion}

The study's findings support the hypothesized existence of multiple interchangeable combinations of risk indicators that equally predict non-recovery from information obtained within days of a traumatic event. Specifically, before cross validation, we identified 789 minimal sets of variables (MBs) that rendered all others non-significant predictors of non-remitting PTSD. The average number of MBs per cross validation trial was 800 . This minor difference is expected, given use of slightly different datasets (i.e., total dataset vs. randomly selected sets of $90 \%$ of the observations).

The existence of such large number of MBs may reflect the presumed multi-causal and equifinal etiology of post-traumatic morbidity, which posits many interchangeable contributing factors and many causal pathways. It is also in line with prior evidence of multiplicity of distinct risk indicators of PTSD [7,12,38].

Our finding extends previous work by translating the previously demonstrated multiplicity of risk indicators into versatile predictive model that can accommodate an array of traumatic situations where one or several known predictors is either unavailable or not contributing significantly. From a practical point of view, such multiplicity points to the potential usefulness of data-informed algorithmic prediction tools to future risk assessments.

This work also extends the array of risk indicators identified by earlier studies: Former studies uncovered salient predictors within large groups, whereas this work demonstrated the ability of less consistently predictive, or less frequently recorded features (e.g., expressing a need for help, or ED length of stay) to carry important information. This underscores ML ability to not to reject

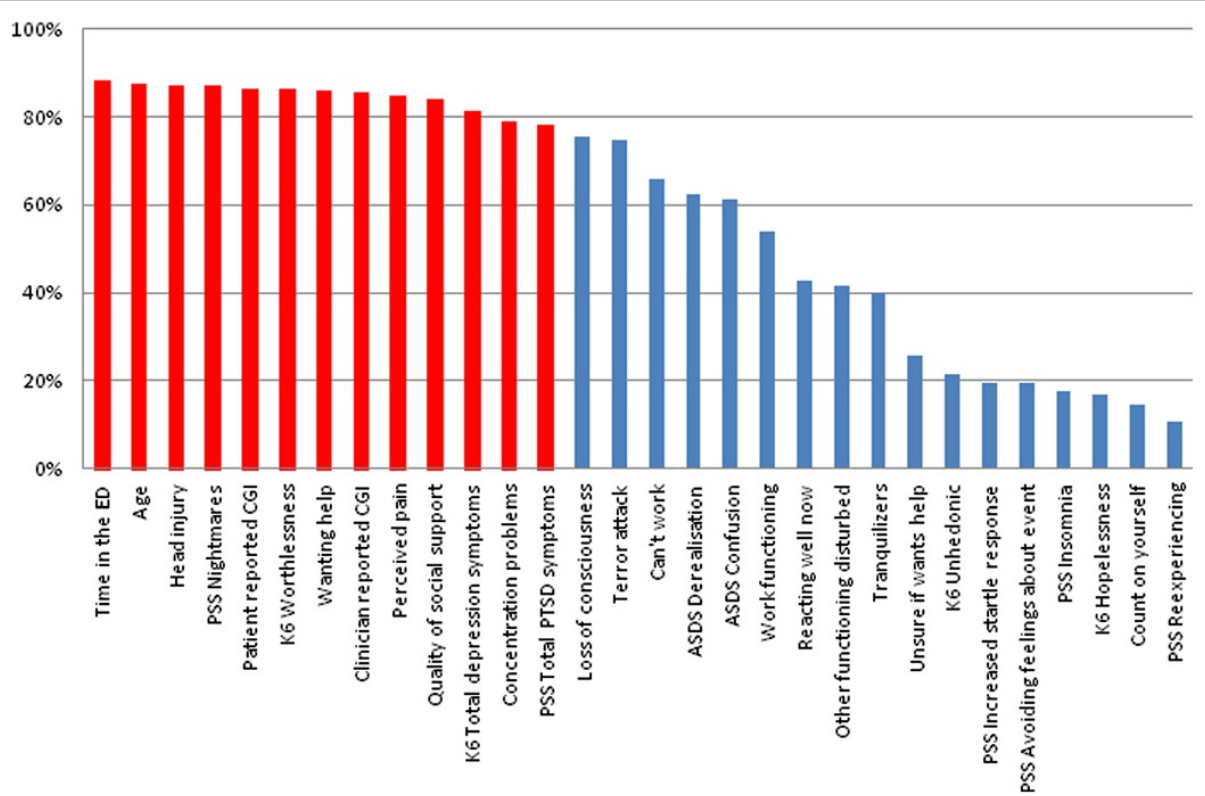

Figure 2 Feature's presence in repeated cross validation trials. The figure shows the frequency (percentage of all trials) in which individual features participate in MBs identified during successive cross-validations trials (only features participating in $>10 \%$ of the trials are presented). Bars in red indicate features selected in $>75 \%$ of cross validation runs $(n=13)$. 
features that are only weakly, or occasionally correlated with an outcome, and thereby fully extract the informational item of datasets.

Within such multiplicity, however, this study identified a few consistently predictive features (e.g., those included in over $75 \%$ of all MBs). Interestingly, these features comprised, side-by-side, prior variables (e.g., age), event and injury parameters, immediate bodily responses (e.g., ED pain), symptoms (nightmares, loss of concentration, total PTSD and depression symptoms), clinicians' observations (e.g., CGI) and more elaborated subjective responses (need for help, sense of worthlessness). Surprisingly, gender was not among the consistent predictors. This might reflect the nature of traumatic events evaluated for this study, most of which were road traffic accidents and thus not gender specific.

MB's predictive accuracy (AUC $=.75$ ) does not support a robust prediction from early information collected. This may illustrate the limited predictive power of data features available for this study, all collected within ten days of a traumatic event. Within such limitations, the results of this work still fare well on two accounts: They firstly show the already reasonable ability of simple, non-invasive, inexpensive observations to predict posttraumatic morbidity. They additionally establish the usefulness of data features that are regularly collected in ED situations. Indeed, this work was not meant to show superior predictive performance, but rather to establish, within the predictive power of a dataset, ways to increase predictive versatility.

Predicting from very early features is also limited in that early symptoms are ubiquitous whereas subsequent morbidity is less frequent (17\% non-remitting in this work). Previous work has shown better prediction of chronic PTSD by data collected one month after the traumatic event [39]. Moreover, other known risk indicators that might become available within days of traumatic exposure (e.g., childhood trauma [40], lifetime mental disorders [41], ED stress hormones [42], gene variants (e.g., FKBP5 [43]), or ED gene expression profiles [12,44]) have not been assessed in this study. Expanding the array of early predictive features by collecting such data might improve the predictive accuracy of early observations.

An important remaining question is the added contribution of putative biomarkers to prediction from of non-invasive, easily retrievable clinical data: Clinical manifestations may express the compounded effect of underlying biological vulnerability and thus might constitute more easily obtainable, non-invasive proxy variables of the latter. Because ML methods can accommodate multimodal information they might help establishing such 'proxy' relationships.

Our results are far from exhausting the potential of machine learning to forecast PTSD. Following similar progress in other areas of medicine [45], ML approaches for forecasting post-traumatic morbidity must be extended and enriched using other data sets and adding other putative predictors. One of many scenarios of such future use of ML decision support algorithms is illustrated in Figure 3. The figure shows how cumulative knowledge of predictive MBs can progressively enrich knowledge-informed algorithmic approach for risk assessment.

\section{Conclusions}

By providing greater versatility, ML-informed algorithms may better identify individuals at risk for post-traumatic morbidity under varying traumatic circumstances. ML capacity to accommodate multimodal information offers new heuristic for forecasting post-traumatic morbidity.

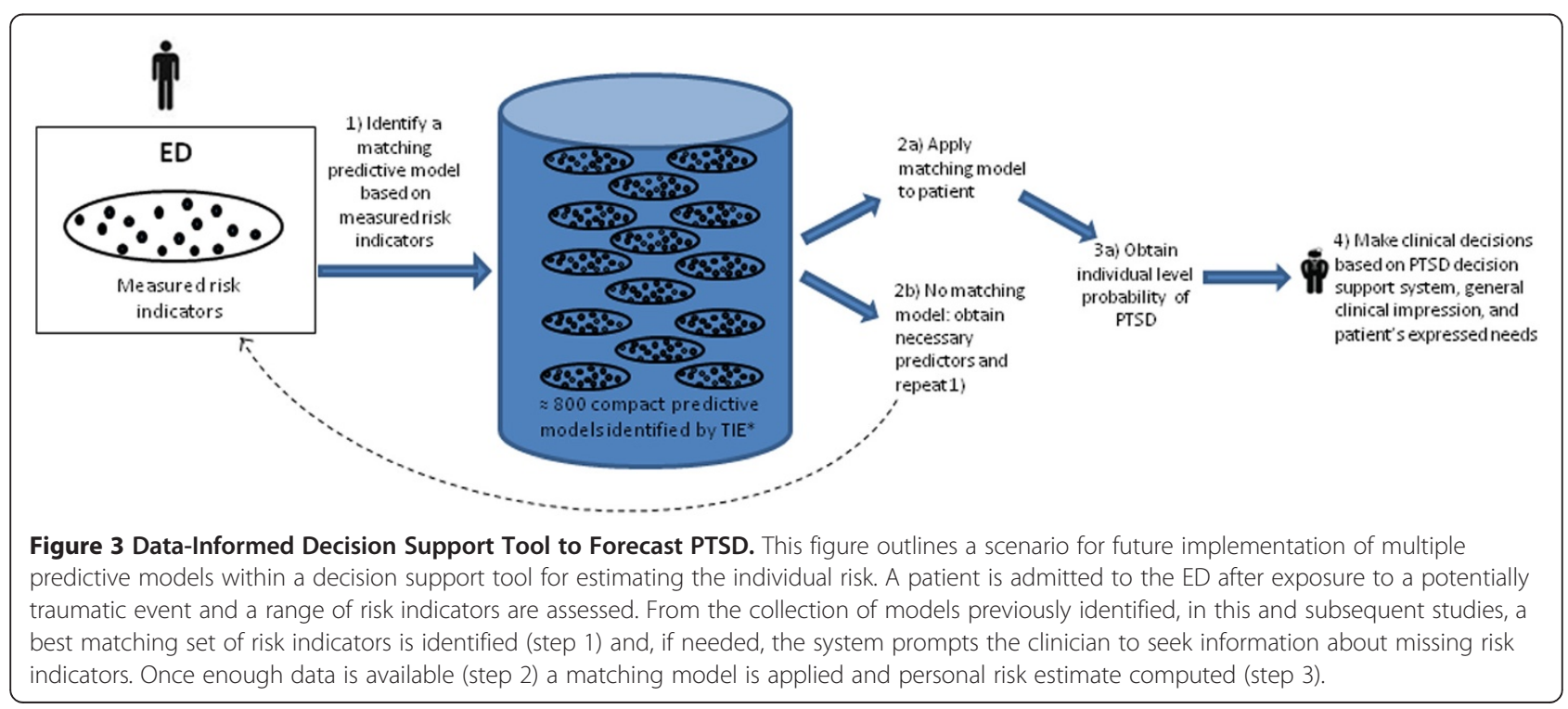




\section{Additional file}

\section{Additional file 1: Glossary.}

\section{Competing interests}

The authors declare that they have no competing interests.

\section{Authors' contributions}

K-I.K, carried out the data analyses for this paper and took the lead on writing the manuscript. I, R. G-L, participated in the data analytic work and in drafting and editing the manuscript. A. Statnikov implemented and adapted data-analytic tools, overviewed the data analytic work and participated in writing the manuscript. Z.L. Participated in data analytic work. A.Y.S. designed the study, lead its implementation and related data collection, quality assurance and management, and participated in designing the current analytic approach in writing of the manuscript. All authors read and approved the final manuscript.

\section{Acknowledgments}

We acknowledge the help of Wei Qi, MD, in editing the manuscript. The following members of the Jerusalem Trauma Outreach and Prevention (J-TOPS) study group performed the 2003-2007 "Prevention of PTSD" study from which the current study data is drawn: Yael Ankri, M.A.; Sara Freedman, Ph.D.; Rhonda Addesky, Ph.D.; Yossi Israeli - Shalev, M.A.; Moran Gilad, M.A; Pablo Roitman M.D.

\section{Source of funding}

US Public Health Service/NIMH research grants \# RO1MH071651 and R34MH102449 to AYS. I R Galatzer-Levy is supported by an NIMH grant $\mathrm{K} 01 \mathrm{MH} 102415$.

\section{Author details School of Medicine, New York, NY, USA. \\ Received: 24 April 2014 Accepted: 23 January 2015 \\ Published online: 16 March 2015}

${ }^{1}$ Department of Psychiatry, NYU School of Medicine, New York, NY, USA. ${ }^{2}$ Research and Knowledge Centre, Danish Veteran Centre, Garnisonen 1, 4100 Ringsted, Denmark. ${ }^{3}$ Center for Health Informatics and Bioinformatics, NYU School of Medicine, New York, NY, USA. ${ }^{4}$ Department of Medicine, NYU

\section{References}

1. Brewin CR, Andrews B, Valentine JD. Meta-analysis of risk factors for posttraumatic stress disorder in trauma-exposed adults. J Consult Clin Psychol. 2000;68(5):748-66.

2. Ozer EJ, Best SR, Lipsey TL, Weiss DS. Predictors of posttraumatic stress disorder and symptoms in adults: A meta-analysis. Psychol Bull. 2003;129(1):52-73.

3. Kessler RC, Sonnega A, Bromet E, Hughes M, Nelson C. Post-traumatic stress disorder in the National Comorbidity Survey. Arch Gen Psychiatry. 1995:52:1048-60

4. Marmar CR, Weiss DS, Schlenger WE, et al. Peritraumatic dissociation and posttraumatic stress in male Vietnam theater veterans. Am J Psychiatry. 1994;151(6):902-7.

5. Shalev AY, Peri T, Canetti L, Schreiber S. Predictors of PTSD in injured trauma survivors: a prospective study. Am J Psychiatry. 1996;153(2):219-25.

6. Forbes D, Creamer M, Biddle D. The validity of the PTSD checklist as a measure of symptomatic change in combat-related PTSD. Behav Res Ther. 2001;39(8):977-86

7. Bryant RA, Harvey AG, Guthrie RM, Moulds ML. A prospective study of psychophysiological arousal, acute stress disorder, and posttraumatic stress disorder. J Abnorm Psychol. 2000;109(2):341-4.

8. Difede J, Barocas D. Acute intrusive and avoidant PTSD symptoms as predictors of chronic PTSD following burn injury. J Trauma Stress. 1999;12(2):363-9.

9. Kleim B, Ehlers A, Glucksman E. Early predictors of chronic post-traumatic stress disorder in assault survivors. Psychol Med. 2007:37(10):1457-67.

10. Mellman TA, David D, Bustamante V, Fins Al, Esposito K. Predictors of post-traumatic stress disorder following severe injury. Depress Anxiety. $2001 ; 14(4): 226-31$.
11. Shalev AY, Peri T, Brandes D, Freedman S, Orr SP, Pitman RK. Auditory startle response in trauma survivors with posttraumatic stress disorder: a prospective study. Am J Psychiatry. 2000;157(2):255-61.

12. Segman RH, Shefi N, Goltser-Dubner T, Friedman N, Kaminski N, Shalev AY. Peripheral blood mononuclear cell gene expression profiles identify emergent post-traumatic stress disorder among trauma survivors. Mol Psychiatry. 2005;10(5):500. - 513, 425.

13. Koenen KC, Stellman JM, Stellman SD, Sommer Jr JF. Risk factors for course of posttraumatic stress disorder among Vietnam veterans: a 14-year follow-up of American Legionnaires. J Consult Clin Psych. 2003;71(6):980-6.

14. Shalev AY, Segman RH. Commentary: biological findings in PTSD - too much or too little? Prog Brain Res. 2008;167:187-99.

15. Orrù G, Pettersson-Yeo W, Marquand AF, Sartori G, Mechelli A. Using support vector machine to identify imaging biomarkers of neurological and psychiatric disease: a critical review. Neurosci Biobehav Rev. 2012;36(4):1140-52.

16. Norman SB, Stein MB, Dimsdale JE, Hoyt DB. Pain in the aftermath of trauma is a risk factor for post-traumatic stress disorder. Psychol Med. 2008:38(4):533-42.

17. Bryan CJ, Clemans TA. Repetitive traumatic brain injury, psychological symptoms, and suicide risk in a clinical sample of deployed military personnel. JAMA Psychiatry (Chicago, III). 2013;70(7):686-91.

18. Haagsma JA, Ringburg AN, van Lieshout EM, van Beeck EF, Patka P, Schipper IB. Prevalence rate, predictors and long-term course of probable posttraumatic stress disorder after major trauma: a prospective cohort study. BMC Psychiatry. 2012;12:236.

19. Bienvenu OJ, Gellar J, Althouse BM, Colantuoni E, Sricharoenchai T, Mendez-Tellez PA, et al. Post-traumatic stress disorder symptoms after acute lung injury: a 2-year prospective longitudinal study. Psychol Med. 2013;43(12):2657-71.

20. Statnikov A, Lytkin NI, Lemeire J, Aliferis CF. Algorithms for Discovery of Multiple Markov Boundaries. J Mach Learn Res. 2013;14:499-566.

21. Breiman L. Statistical modeling: the two cultures. Stat Sci. 2001;16(3):199-215.

22. Statnikov A, Aliferis CF. Analysis and computational dissection of molecular signature multiplicity. PLoS Comput Biol. 2010;6(5):e1000790.

23. Galatzer-Levy IR, Karstoft KI, Statnikov A, Shalev AY. Quantitative forecasting of PTSD from early trauma responses: a Machine Learning application. J Psychiatr Res. 2014;59:68-76.

24. Boser BE, Guyon IM, Vapnik VN. A training algorithm for optimal margin classifiers. In: Proceedings of the fifth annual workshop on Computational learning theory (COLT '92). New York, NY, USA: ACM; 1992. 144-152. doi:10.1145/130385.130401

25. Galatzer-Levy IR, Ankri Y, Freedman S, Israeli-Shalev Y, Roitman P, Gilad M, et al. Early PTSD Symptom Trajectories: Persistence, Recovery, and Response to Treatment: Results from the Jerusalem Trauma Outreach and Prevention Study (J-TOPS). PLoS One. 2013;8(8):e70084.

26. Shalev AY, Ankri Y, Israeli-Shalev Y, Peleg T, Adessky R, Freedman S. Prevention of posttraumatic stress disorder by early treatment: results from the Jerusalem Trauma Outreach And Prevention study. Arch Gen Psychiatry. 2012;69(2):166-76.

27. Shalev AY, Ankri YL, Peleg T, Israeli-Shalev Y, Freedman S. Barriers to receiving early care for PTSD: results from the Jerusalem trauma outreach and prevention study. Psychiatr Serv. 2011;62(7):765-73.

28. Foa EB, Tolin DF. Comparison of the PTSD Symptom Scale-Interview Version and the Clinician-Administered PTSD scale. J Trauma Stress. 2000;13(2):181-91.

29. Kessler RC, Andrews G, Colpe LJ, Hiripi E, Mroczek DK, Normand SL, et al. Short screening scales to monitor population prevalences and trends in non-specific psychological distress. Psychol Med. 2002;32(6):959-76.

30. Bryant RA, Moulds ML, Guthrie RM. Acute Stress Disorder Scale: a self-report measure of acute stress disorder. Psychol Assess. 2000;12(1):61-8.

31. Guy W. Clinical global impression scale. The ECDEU Assessment Manual for Psychopharmacology-Revised Volume DHEW Publ No ADM 76. 1976;338:218-22.

32. Foa EB, Ehlers A, Clark DM, Tolin DF, Orsillo SM. The Posttraumatic Cognitions Inventory (PTCl): Development and validation. Psychol Assess. 1999;11(3):303-14.

33. Lazarus RS, Folkman S. Stress, Appraisal, and Coping. New York: Springer; 1984

34. Bryant RA, O'Donnell ML, Creamer M, McFarlane AC, Silove D: A Multisite Analysis of the Fluctuating Course of Posttraumatic Stress Disorder. JAMA psychiatry (Chicago, III.). Jun 19 2013:1-8

35. Statnikov A, Tsamardinos I, Brown LE, Aliferis CF. Causal Explorer: A Matlab Library of Algorithms for Causal Discovery and Variable Selection for 
Classification. In: Guyon IM, Aliferis CF, Cooper GF, editors. Challenges in Machine Learning Volume 2: Causation and Prediction Challenge. Bookline, Massachusetts: Microtome Publishing; 2010. p. 267-78.

36. Chang C-C, Lin C-J, LIBSVM. A library for support vector machines. ACM Trans Intell Syst Technol. 2011;2(3):1-27.

37. Bradley AP. The use of the area under the ROC curve in the evaluation of machine learning algorithms. Pattern Recogn. 1997;30(7):15.

38. Shalev AY, Freedman S. PTSD following terrorist attacks: a prospective evaluation. Am J Psychiatry. 2005;162(6):1188-91.

39. Freedman SA, Brandes D, Peri T, Shalev A. Predictors of chronic post-traumatic stress disorder. A prospective study. Br J Psychiatry. 1999;174:353-9.

40. Breslau N, Chilcoat HD, Kessler RC, Davis GC. Previous exposure to trauma and PTSD effects of subsequent trauma: results from the Detroit Area Survey of Trauma. Am J Psychiatry. 1999;156(6):902-7.

41. Koenen KC, Harley R, Lyons MJ, Wolfe J, Simpson JC, Goldberg J, et al. A twin registry study of familial and individual risk factors for trauma exposure and posttraumatic stress disorder. J Nerv Ment Dis. 2002;190(4):209-18.

42. Shalev AY, Videlock EJ, Peleg T, Segman R, Pitman RK, Yehuda R. Stress hormones and post-traumatic stress disorder in civilian trauma victims: a longitudinal study. Part I: HPA axis responses. Int J Neuropsychopharmacol. 2008;11(03):365-72.

43. Binder EB, Bradley RG, Liu W, Epstein MP, Deveau TC, Mercer KB, et al. Association of fkbp5 polymorphisms and childhood abuse with risk of posttraumatic stress disorder symptoms in adults. JAMA. 2008;299(11):1291-305.

44. Boscarino JA, Erlich PM, Hoffman SN, Zhang X. Higher FKBP5, COMT, CHRNA5, and CRHR1 allele burdens are associated with PTSD and interact with trauma exposure: implications for neuropsychiatric research and treatment. Neuropsychiatr Dis Treat. 2012;8:131-9.

45. Visweswaran S, Angus DC, Hsieh M, Weissfeld L, Yealy D, Cooper GF. Learning patient-specific predictive models from clinical data. J Biomed Inform. 2010;43(5):669-85.

\section{Submit your next manuscript to BioMed Central and take full advantage of:}

- Convenient online submission

- Thorough peer review

- No space constraints or color figure charges

- Immediate publication on acceptance

- Inclusion in PubMed, CAS, Scopus and Google Scholar

- Research which is freely available for redistribution 